\title{
ENHANCING NUCLEAR POWER PLANT PERFORMANCE THROUGH THE USE OF ARTIFICIAL INTELLIGENCE
}

DEPARTMENT OF ENERGY CONTRACT \# DE-FG07-88ER12824

PROGRAM MANAGER: ROBERT E. UHRIG

PRINCIPAL INVESTIGATORS: MICHAEL JOHNSON

ALIANNA MAREN

LAURENCE MILLER

ROBERT UHRIG

BELLE UPADHYAYA

\author{
JUNE 15, 1989
}

DEPARTMENT OF NUCLEAR ENGINEERING

UNIVERSITY OF TENNESSEE

KNOXVILLE, TENNESSEE 
THE UNIVERSITY OF TENNESSEE

KNOXVILLE

June 15, 1989

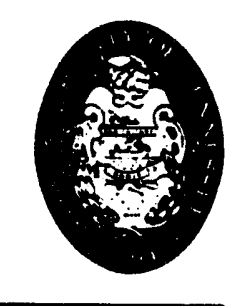

College of Engineering

Department of juclear Engineering
Dr. David Woodall

DOE Co-Technical Monitor

EG\&G Idaho, Inc.

Idaho National Engineering Laboratory

P. O. Box 1625

Idaho Falls, ID 83415

Dr. John Lewellan

DOE Co-Technical Monitor

NE-462, Germantown

U.S. Department of Energy

Washington, D.C. 20585

Dear Dr. Lewellan:

Subject: First Annual Report on U.S. DOE Contract \# DOE-DEFG88ER12824, entitled "Enhancing Nuclear Power Plant Performance through the Use of Artificial Intelligence."

Enclosed is the first annual report for the period september 30, 1988 to May 31, 1989 on the above referenced contract. It consists of an "Overview" of the progress made in the various projects, along with a several supporting internal reports that describe the work in considerable detail. In several cases, papers have been published or submitted for publication are referenced. Copies of these reports are also attached. Also, two papers describing what may be "patentable ideas" were submitted on June 1, 1989 to DOE patent counsel in the DOE Chicago Operations office for patent review. These reports will be incorporated into a later report when the patent review has been completed and appropriate action taken (i.e., a patent application is submitted or a decision is made not to submit an application).

As can be seen from the attached report, this program is off to an excellent start. Host activities have progressed more rapidly than expected, and papers describing this work (with acknowledgement of the support of DOE under this contract) are beginning to appear in the pertinent national and international technical literature. Twelve graduate students from four departments are working on this program 
Dr. David Woodall
Dr. John Lewellan

under the direction of five UT professors. Never-the-1ess, certain activities have not progressed as rapidly as expected. A reassessment of the various tasks has resulted in several changes in the second-year program, including terminating one task and replacing it with another. These changes will be described in the proposal for continuation of this work during FY -90 .

We are very appreciative of the support provide by the Department of Energy and look forward to continued progress during the next two years.

sincerely yours,

Robert E. Uhrig

Distinguished Professor of Engineering

Enclosures: See attached list 
Dr. David Woodall

Page 3

June 15,1989

Dr. John Lewellan

\section{IIST OF ENCIOSURES}

OVERVIEW: Enhancing Nuclear Power Plant Performance through the Use of Artificial Intelligence.

TASK 1: MUITI-SOURCE INFORYATION FUSION THROUGH ADAPTIVE NAURAL NETWORKS

A. A. J. Maren and I. A. Pereira, Multisensor Information Fusion: A comprehensive literature Review," Internal Report, May 1989.

B. A. J. Maren, "Multisensor Information Fusion: a Contribution to the Phase I Final Report, Intelligent Control for Nuclear Power Plants," Internal Report, June 1989.

C. A. J. Maren, R. M. Pap, and C. T. Harston, "A Hierarchical Structure Approach to Multi-sensor Information Fusion," Presented to the Second National symposium on Sensors and Sensor Fusion," Orlando, FL, March 27, 1989.

D. A. J. Maren, R. M. Pap, and C. T. Harston, "A Hierarchical Structure Approach to Multi-sensor Information Fusion," Proceedings of the SPIE Technical Symposium on Aerospace Sensing," Orlando, FL, March 28-31, 1989.

TASK 2: APPLICATION OF NEURAL NETWORKS TO IN-CORE FUEL MANAGEYENT

E. R. E. Uhrig and L. F. Miller, "Use of Neural Networks for InCore Fuel Management," ANS Session on "Expert systems in the Fuel cycle", Transactions of the American Nuclear society, Vol. 59, June 4-8, 1989.

F. L. F. Miller, M. Abdalla, and R. E. Uhrig, "An Application of Neural Networks to In-Core Fuel Management," UTK Internal Report, June 1989.

\section{TASK 3: SENSOR VALIDATION USING NEURAI NETMORKS}

G. B. R. Upadhyaya, E. Eryurek, and G. Mathai, MApplication of Neural Computing Paradigms for signal Validation, " Proceedings of the Seventh Power Plant Dynamics, Control and Testing Symposium, vol. 1, pp 227.01-27.18, Knoxville, Tw, May 15-17, 1989. 
Dr. David Woodall

Page 4

June 15,1989

Dr. John Lewellan

H. E. Eryurek and B. R. Upadhyaya, "Sensor Validation in Power Plants using Adaptive Backpropagation Neural Networks," To be presented at the 1989 IEEE Nuclear science Symposium, san Francisco, October 25-27, 1989.

I. B. R. Upadhyaya and E. Eryurek, "Development and Application of the Backpropagation Network for Sensor Validation." UTK Internal Report, May 1989.

TASK 4: DIAGNOSIS OF NUCLEAR POWER PIANT MALFUNCTIONS AND FAIIURES

J. R. E. Uhrig, "Use of Neural Networks in Nuclear Power Plants," Proceedings of the 7 th Power Plant Dynamics, Control Testing Symposium, vol. 1, pp 26.01-26.05, Knoxville, Tennessee, May 15-17, 1989.

K. R. E. Uhrig, "Use of Neural Networks in Nuclear Power Plant Diagnostics," Proceedings of the "International Conference on Availability Improvements in Nuclear Power Plants," Madrid, Spain, April 10-14, 1989, (In press) 1989.

I. R. E. Uhrig and Z. Guo, "Use of Neural Networks in Expert Systems for Nuclear Power Plant Diagnostics," Proceedings of "Applications of Artificial Intelligence VII," SPIE Technical Symposium on Aerospace Sensing, Orlando, Florida, March 28$30,1989$.

M. E. B. Bartlett, "Neuro-Connectionist Learning Techniques, Learning Generalization, and Applications," UTK Internal Report, June 1989.

N. Zhichao Guo, "Use of Neural Network in an Expert system to Enhance Nuclear Power Safety." UTK Internal Report, April 1989.

TASK 5: KNOWIEDGE ACOUISITION

No Publications.

TASK 6: JOINT TVA UT PROJECTS FOR NUCIEAR OPERATION

0. J. A. Boshers, "SAFETY REVIEW ADVISOR: An Expert system to Aid Performance of 10CFR50.59 Evaluations," Internal Report, May 1989. 
Dr. David Woodall

Dr. John Lewellan

P. J. A. Boshers and I. F. Alguindigue, "The safety Review Advisor: An Expert System to Evaluate Proposed Modifications to Nuclear Power Plants," (Summary only). American Nuclear Society Eastern Regional student Conference, Knoxville, IN, March 25, 1989.

Q. J. A. Boshers, I. E. Alguindigue, C. G. Burnett, and R. Z. Uhrig, "Safety Review Advisor" (Poster presentation), EPRI Conference on "Expert Systems Applications for the Electric Power Industry," Orlando, FL, June 5-8, 1989. 
FIRST ANNUAL REPORT

\section{ENHANCING NUCLEAR POWER PLANT PERFORMANCE THROUGH THE USE OF ARTIFICIAL INTELLIGENCE}

DEPARTMENT OF ENERGY CONTRACT \# DE-FG07-88ER12824

PROGRAM MANAGER: ROBERT E. UHRIG

PRINCIPAL INVESTIGATORS: MICHAEL JOHNSON

ALIANNA MAREN

LAURENCE MILLER

ROBERT UHRIG

BELLE UPADHYAYA

JUNE 15, 1989

DEPARTMENT OF NUCLEAR ENGINEERING

UNIVERSITY OF TENNESSEE

KNOXVILLE, TENNESSEE 
Introduction..............................

objective................................

Task 1: Multi-source Information Pusion through the

Use of Adaptive Neural Network Computations.....4

Task 2: Application of Neural Networks to In-Core

Nuclear Fuel Management.................6

Task 3: Sensor Validation using Neural Networks........9

Task 4: Diagnosis of Impending and Actual Plant

Failures.........................12

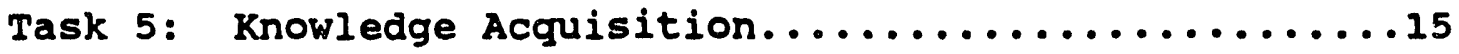

Task 6: Joint UT/TVA Projects for Nuclear operation....17

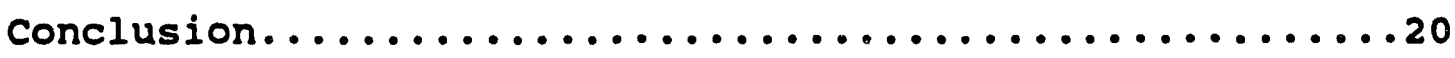

Appendicies..............................21 
OVIRVTIN

ENHANCING NUCLEAR POKER PIANT PERFORYANCE

THROUGH THE USE OF ARTIFICIAL INTELIIGENCE

Introduction. In the summer of 1988 , the Department of Nuclear Engineering (NE) at the University of Tannessee (UT) in Knoxville was selected to carry out a research program in winhancing the Operation of Nuclear Power plants through the use of Artificial Intelligence." This program is sponsored by the Department of Energy's Office of Energy Research under 10CFR605 for Nuclear Engineering Research.

objective. The objective of the research is to advance the state-of-the-art of nuclear power plant control, safety, management, and instrumentation systems through the use of artificial intelligence (AI) techniques, including both expert systems and neural networks. Tre emphasis will be placed on methods that can be implemented on a rapid or real-time basis. A second, but equally important, objective is to build a broadly based critical mass of expertise in the artificial intelligence field that can be brought to bear on the technology of nuclear power plants. Both of these goals are being met. This overview and the attached technical reports describe the work that is being carried out. Although in some cases, the scope of the work differs somewhat from the specific tasks described in the original proposal, all activities are clearly within the overall scope of the contract.

Direction of the research on the Department of Energy contract, is shared among the following UT faculty:

Dr. Michael Johnson, Dept. of Psychology, UT Knoxville

Dr. Alianna Maren, Dept. of Computer Science, UT Space Inst.

Dr. Laurence Miller, Dept. of Nuclear Engr., UT Knoxville

Dr. Robert Uhrig, Dept of Nuclear Engr. , UT Knoxville

Dr. Belle Upadhyaya, Dept. of Nuclear Engr., UT Knoxville

The following graduate students are involved in the artificial intelligence research activities supported during FY-89 by the DOE contract and the UT Science Alliance (a UT/ORNL program that supports the Program Manager's faculty appointment): Although these students are enrolled in degree programs in four UT departments, all their work concerns applications to nuclear plants. 
Name

Israel Alguindigue

Eric Bartlett

Alfred Boshers

Roberta Dewick

Evren Eryurek

Aouatef Gacem

James Golden

Zhichao Guo

Abdal la Mohamed

Magid Natour

Lisa Pereira

Iouis Qualls

$\begin{array}{llll}\text { Level } & \text { Dept. } & \text { Institution } & \text { Advisor } \\ \text { Ph.D. } & \text { C.S. } & \text { UT Knoxville } & \text { Uhrig } \\ \text { Ph.D. } & \text { N.E. } & \text { UT Knoxville } & \text { Uhrig } \\ \text { M.S. } & \text { N.E. } & \text { UT Knoxville } & \text { Uhrig } \\ \text { Ph.D. } & \text { Prych. } & \text { UT Knoxville } & \text { Johnson } \\ \text { M.S. } & \text { N.E. } & \text { UT Knoxville } & \text { Upadhyaya } \\ \text { Ph.D. } & \text { E.E. } & \text { UT Knoxville } & \text { Trevedi/Uhrig } \\ \text { M.S. } & \text { C.S. } & \text { UT Space Inst. } & \text { Maren } \\ \text { Ph.D. } & \text { N.E. } & \text { UT Knoxville } & \text { Unrig } \\ \text { Ph.D. } & \text { N.E. } & \text { UT Knoxville } & \text { Miller } \\ \text { Ph.D. } & \text { N.E. } & \text { UT Knoxville } & \text { Katz/Uhrig } \\ \text { M.S. } & \text { C.S. } & \text { UT Space Inst. Maren } \\ \text { M.S. } & \text { N.E. } & \text { UT Knoxville } & \text { Uhrig }\end{array}$

The following six general research topics were described in the proposal submitted to the DOE last year:

1. Multi-Source Information Fusion through Adaptive Neural Network Computations (Maren)

2. Application of Neural Networks to In-Core Fuel Management (Miller)

3. Sensor Validation (Upadhyaya)

4. Diagnosis of Impending and Actual Plant Failures (Uhrig)

5. Knowledge Acquisition (Johnson)

6. Joint TVA/UT Projects for Nuclear Operation (Uhrig/TVA Staff)

Progress has been made in all of these areas. Perhaps the most significant progress has been in the application of neural networks in the first four projects. In order to bring the graduate students "up to speed" in the technology of neural networks, the Program Manager (Uhrig) taught a course on neural networks during the Fall semester (August through December) 1988. All graduate students at UT Knoxville working on this project who expected to use neural networks in their research were required to attend and participate in the class. In lieu of a final examination, each student was required to prepare a special project using neural netwurks. Students working under this contract were encouraged to prepare projects that relate to this contract.

Two commercial computer programs for building neural networks on personal computers were made available to the students in this class and to those working on this program: "NEURALWARE" by NeuxalWorks Inc. and "ANSIM" by Scientific Applications International Corporation. Both of these programs run on "AT class" personal computers. However, many of the students elected to write their own computer program for the neural networks, usually for the MicrovAX-VAXstation 2000 network in the Nuclear Engineering Department which is tied by phone line to the University VAX computers, because of the greater computing power of the VAX computers. 
An overview of each task and the progress in each of the related projects is presented here. An introduction is given in each case to present the context in which the research is related to the objective of the overall objectives of the program. 
TASK 2: MULTI-SOURCE INFORYATION FUSION

THROUGH THE USE OF ADAPTIVE NEURAI NETWORK COYPUTATIONS (YAREN)

Thousands of sensors are used to monitor the safety and operations of nuclear power plants. The number of sensors, and the complexity of the processes that they monitor, makes it very difficult for humans to understand what is happening in a nuclear power plant just by observing sensor readouts. In order to interpret the output from these many sensors, we have to develop intelligent syatems that can aid the human operators. Although these intelligent systems ultimately are intended to provide a means of "controliing" complex processes, only "open loop" systems that provide information and advice to operators are being considered for nuclear power plants at the present time.

Intelligent systems must be able to:

- Fuse and compress information from many sensors,

- Validate the output of different sensors,

- Recognize the patterns of events portrayed by sensor outputs,

- Interpret the meaning of the different patterns, and

- Interact with the human operators of the power plant, to provide them with the information that they want and need in a way that it can be understood.

The technical issues in intelligent control can be divided into three levels: the low-level processing must combine and compress sensor data and must validate sensor performance; the mid-level processing must perform pattern recognition; and the high-level processing must interpret the patterns, or assign meaning to them and communicate that meaning to the operators.

So far in this task, the work has been limited to fusing (combining) the information from several sources (or sensors of different types), commonly called multi-source information fusion (MSIF). The method uses "hierarchical data structures" (HDSs--data records that successively contain less detail) to organize the sensor monitored events. By abstracting (removing details) sensor data to the "structure" level, the combining of sensor information and the interpretation of patterns of complex events is made easier. The principal thrust of this task to date has been to develop a cooperative/competitive neural network method of creating and combining HDSs (after they have been reduced to the "gtructuren level) and to developing a method of reducing the dimensionality (complexity) of multi-sensor data.

It is expected that the sensor fusion take place at the structure level. By fusing or combining HDSs from individual sensors, it is possible to create a new Multisensor-fused HDS (MIDS) that contains information extracted from the HDSs, resulting in a further data compression as well as fusion. 
Progress. During the first six months on this program, coding and testing for a computer program which extracts events from a data stream and creates a Hierarchical Data structure represented the temporal organization of these events has been completed. Concepts for sensor data fusion, temporal pattern recognition, and the use of connectionist expert systems for intelligent pattern interpretation have also been developed, but not implemented.

During the past eight months, the following reports dealing with this work were prepare and are included as appendices. The preparation of the last two reports was partially supported by another Government agency.

A. J. Maren and L. A. Pereira, Myultisensor Information Fusion: A comprehensive literature Review," Internal Report, May 1989.

A. J. Maren, "Multisensor Information Fusion: a Contribution to the Phase I Final Report, Intelligent Control for Nuclear Power Plants," Internal Report, June 1989.

A. J. Maren, R. M. Pap, and C. T. Harston, "A Hierarchical Structure Approach to Multi-sensor Information Fusion," Presented to the Second National Symposium on Sensors and Sensor Fusion," Orlando, $F L$, March $27,1989$.

A. J. Maren, R. M. Pap, and C. T. Harston, "A Hierarchical Structure Approach to Multi-sensor Information Fusion, "Proceedings of the SPIE Technical Symposium on Aerospace Sensing," Orlando, FL, March $28-31,1989$. 


\section{TASK 2: APPILCATION OF NEURAL NETWORKS TO IN-CORE NUCLEAR FUEI MANAGEYFNT (YILIFR)}

There are over one-hundred nuclear power reactors in the United States that will continue to operate for several more decades. More that 5000 new fuel asaemblies are required to fuel these reactors each year, and several billion dollars of expenditures are required each year for the fuel. If a one percent improvement in fuel utilization is obtained, a savings of approximately 50 million dollars could be realized on an annual basis. Thus, there is a strong financial motivation for improving the utilization of nuclear fuel. This work evaluates the feasibility of using neural networks to enhance the stateof-the-art for in-core fuel management.

There are two general objectives of this research that, if accomplished, will advance practical (as well as academic) capabilities in the area of in-core fuel management. Briefly stated thoy are:

1. Develop a neural network that captures the nonlinear behavior of fuel depletion. This network will predict end-of-cycle (EOC) conditions, as well as intermediate-cycle conditions, based of beginning-of-cycle (BOC) specifications.

2. Develop a neural network that will predict BOC specifications that correspond to desired EOC conditions.

This study examines the feasibility of using neural networks for performing scoping fuel depletion evaluations, which will permit very rapid and exhaustive optimization studies. Thus, the networks under development will be useful for survey calculations (and for twodimensional optimization studies) to obtain optimal candidate reload cores. Conventional, three-dimensional, state-of-the-art depletion calculations still will be required to meet licensing requirements.

The first task of this endeavor was to obtain training data in the form of neutronics calculations and of actual plant operating data from the in-core fuel management group with the Tennessee valley Authority. The calculations are two-dimensional scoping calculations that extend over $s i x$ reload cycles, and the operating data are from the first three cycles of TVA's Sequoyah Unit 1 Nuclear Power Plant. The calculated results include relative power and exposure for each assembly for eleven time steps during reload intervals. Some of the details relative to enrichment and burnable poison concentrations for each assembly will be made available as the need for these values arises.

Two neural network paradigms have been evaluated for this application. The first paradigm tested utilized a conventional fullyconnected back propagation algorithm applied to a single assembly and to a group of eight assemblies. The second paradigm tested utilized a once-through training algorithm ${ }^{1}$ with linear neuride transfer functions. The software for calculating the network parameters was written by graduate students working on this project. The objective of each of 
these studies was to predict intra-cycle relative powers during a reload cycle while using only a portion of the calculated results for training the network.

Progress. Discrepancies of less than 10 between the network results and the neutronics calculations are obtained for the fullyconnected back propagation network. These results are encouraging, but the accuracy need to be improved for this procedure to be of practical value. In order to do this, essential parameters that define the core loading need to be included as input. Because there are correlations among the typical descriptors, they may not all need to be fully connected into the network. It would require about a cuarter million weights if all neurides were fully connected. Thus, a network structure that utilizes a combination of ubassemblies (or groups of subassemblies) as neurides will be examined. Another logical compromise (but probably not a performance compromise) to the full-connection approach is a "next-door-neighbor" connection network, which is representative of the core physics associated with fuel depletion.

The once-through training algorithm was applied to all of the assemblies in one-fourth of the reactor core to predict intra-cycle values of relative power with total energy yield and the initial relative power distribution as the input variables. This paradigm works well if adequate training data are provided, but it performs poorly if only BOC and EOC conditions are provided as input data. A network of practical value (after it is trained) must predict intra-cycle parameters based only on BOC and EOC specifications. This paradigm (and associated software - as it stands) will probably not satisfy this objective, but it could be enhanced in several ways. Our plans are to associate it with a more sophisticated hidden layer structure that contains the essential fuel assembly depletion dynamics.

It will be necessary to develop computational methods that will identify essential neural network parameters for networks with arbitrarily specified connections and with neurides with arbitrary complexity. It is expected that the connections and the neuride transfer functions will need to be structured on the known physics of the fuel burnup phenomenon. The next phase of our effort will concentrate on developing software and network structures that have inherent capability for capturing the essential nonlinear characteristics of in-core fuel depletion physics.

The current testing of neural network paradigms has involved the use relative power distributions (from neutronics calculations and plant operating data) and average burnup as input and outputs, but more detailed specifications regarding the $B O C$ and $B O C$ conditions will be incorporated into the network configurations as the computational methodologies improve. In particular, enrichment, burnable poisons, location of assembly, type of assembly and exposure history need to be specified for BOC and EOC conditions. 
A preliminary report of this work was solicited by the ANS Fuel Cycle Division for presentation at a Special Session on Fuel Management at the 1989 Spring Meeting of the American Nuclear Society in Atianta, June 4-8, 1989. The summary of this paper, as well as the internal report listed below, are attached as appendices.

R. E. Uhrig and L. F. Miller, wese of Neural Networks in Nuclear Fuel Cycle studies," Transactions of the American Nuclear society, Atlanta, GA, June 1989. (In Press)

I. F. Miller, M. Abdalla, and R. E. Uhrig, "An Application of Neural Networks to In-Core Fuel Management," UTK Internal Report, June 1989.

1. Z. Guo and R. E. Uhrig, "Once-Through Supervised Learning Network," (Private communications). 


\section{TASK 3: SENSOR VALIDATION USTNG NEURAL NETWORKS}

The estimation of system variables is performed traditionaliy using either physical models or empirical models. This information is utilized in control systems for sensor validation and for independent monitoring of slow changes in the process or instrument behavior. The model-base prediction assumes a fixed structure for characterizing steady-state or dynamic relationships among process variables. In application to large-scale complex systems, the determination of highfidelity models is very tedious. Bmpirical steady-state (and quasisteady-state) modeling has been applied successfully for plant variable prediction using small signal sets (generally less than $8 i x$ ). Since the objective here is to associate signals in a subsystem of a plant, such a relationship can be developed using neural network models." Both steady-state and transient behavior can be incorporated into the network during training.

The objective of this task is to develop and apply one or more neural network paradigms for automated sensor validation during both steady-state and transient operations. The use of neural networks for signal estimation has several advantages. It is not necessary to define a functional form relating a set of process variables. The functional form as defined by a neural network system is implicitly nonlinear. Once the network is properly trained, the future prediction can be interpolated in real-time. The state estimation is less sensitive to measurement noise compared to direct model-based techniques. As new information about the system becomes available, the network connection weights can be updated without relearning the entire data set. These and other features of neural networks will be exploited in developing an intelligent system for on-line sensor qualification.

In general, the principle of neural network systems can be used to associate one set of information with another set. The approach for sensor validation using neural networks utilizes one of the multilayer perceptrons, called the backpropagation network (BPN) which is a fully connected hetero-associative network. For signal validation, the input layer neurides correspond to the input signals and the output layer may have just one signal which is to be predicted or estimated. A single middle (or hidden) layer of neurides is used. The implicit relationship is determined through the connection weights. While the number of neurides in the hidden layer is somewhat arbitrary, a larger number increases the fault tolerance and makes the network more robust to increasing noise-to-signal ratios and even 1088 of an input signal. The BPN algorithm computes the weights between pairs of processing elements (in adjacent layers) such that the difference between the actual output and the network output is minimized in a least-squares sense.

This work includes the study of the following aspects of network properties and implementation of the algorithm for sensor validation and pattern classification. 
1. Adaptive variation of the sigmoidal function shape parameter during network training to accelerate convergence. This is referred to as "progressive thresholding."

2. The choice of the number of processing elements in the intermediate layer

3. Determination of an error criterion to quantify prediction performance.

4. Study of interpolation and extrapolation capabilities of the BPN with application to signal estimation.

5. Development of a new BPN algorithm computer software and implementation in a VAX-2000 workstation. This is more versatile than some of the commercial neural network packages available.

Results. The current study and research in neural networks have provided a deeper understanding of the principles of multilayer perceptrons and their ability to classify and associate information for nongaussian parameters and for nonlinear separation of information clusters. The inherent parallelism in the network and its ability to characterize very general cause-and-effect relationships have resulted in the following contributions to this task during the first six months.

1. Development and implementation on a computer-workstation of a new software system using a backpropagation network.

2. Incorporation of adaptive changes in the sigmoidal decision function, thus allowing several orders of magnitude increase in the network training rate.

3. Very fast recall during state estimation requiring a single pass through the network.

4. Application to sensor verification using operational plant start-up data from a PWR and the EBR-II. This, to the best of our knowledge, is the first successful application of neural networks for sensor verification.

5. Very few restrictions on the allowable number of training patterns, and the number of process signals at the input layer.

The software and network architecture is such that other applications can be easily studied using this new computer software system. The results of this phase of the work provide a solid foundation for extending neural computing to process diagnostics and the identification of process component faults. The BPN will be applied to the monitoring of spectral signatures derived from the analysis of process fluctuations (noise signals) in power reactors. The abundance 
of noise signatures results in a large-scale problem, which can be effectively handled using neural networks. The approach described here has high fault tolerance to missing input signals and signal noise corruption.

Work on this task has resulted in two papers accepted for presentation at national conferences, as well as an internal report. These are included as appendices to this report.

B. R. Upadhyaya, E. Eryurek, and G. Mathai, "Application of Neural Computing Paradigms for signal validation," Proceedings of the seventh Power Plant Dynamics, Control and Testing Symposium, vol. 1, pp 227.0127.18, Knoxville, TN, May 15-17, 1989.

E. Eryurek and B. R. Upadhyaya, "Sensor Validation in Power Plants using Adaptive Backpropagation Neural Networks," To be presented at the 1989 IEEE Nuclear Science Symposium, San Francisco, October 25-27, 1989.

B. R. Upadhyaya and E. Eryurek, "Development and Application of the Backpropagation Network for Sensor Validation." UTK Internal Report, May 1989. 


\section{TASK 4: DIAGNOSIS OF IMPENDING AND ACTUAL PLANT FAILURES}

When a complex system plant is operating safely, the outputs of hundreds, or even thousands, of sensors or control room instruments form a pattern (or unique set) of readings that represent a "safe" state of the plant. When a disturbance occurs, the sensor outputs or instrument readings form a different pattern that represents a different state of the plant. This latter state may be safe or unsafe, depending upon the nature of the disturbance. The fact that the pattern of sensor outputs or instrument readings is different for different conditions is sufficient to provide a basis for ldentifying the state of the plant at any given time. To implement a diagnostic tool based on this principle, that is useful in the operation of complex systems, requires a rapid (real-time), efficient method of "pattern recognition." Neural networks offer such a method.

Useful Features of Neural Networks. Neural networks may be designed so as to classify an input pattern as one of several predefined types of faults or transients (e.g., the various fault or transient states of a power plant) or to create, as needed, categories or classes of system states which can be interpreted by a human operator. Neural networks have demonstrated high performance even when presented with noisy, sparse and incomplete data.

A second desirable feature of neural networks is their ability to respond in real-time to the changing system state descriptions provided by continuous sensor input. For complex systems involving many sensors and possible fault types (such as nuclear power plants), real-time response is a difficult challenge to both human operators and expert systems. However, once a neural network has been trained to recognize the various conditions or states of a complex system, it only takes one cycle to detect a specific condition or state. Because neural networks can be trained to recognize the patterns of different sensor outputs or instrument readings that give rise to different system states or faults, they are ideally suited for real-time diagnostics.

Neural networks have the ability to recognize patterns, even when the information comprising these patterns is noisy or incomplete. Unlike most computer programs, neural network implementations in hardware are very fault tolerant; i.e. neural network systems can operate even when some individual nodes in the network are damaged. The reduction in system performance is about proportional to the amount of the network that is damaged. Thus, systems of artificial neural networks have high promise for use in environments in which robust, fault-tolerant pattern recognition is necessary in a real-time mode, and in which the incoming data may be distorted or noisy. This makes artificial neural networks ideally suited as a candidate for fault monitoring and diagnosis, control, and risk evaluation in complex systems, such as nuclear power plants. 
Results. Several individual projects have been initiated under this task. The project that has progressed the farthest is "The Identification of system Transients" which has resulted in presentations and publication in the proceedings of three papers, two at national meetings and one at an international meeting.

This project and several related activities are described below. At this time, these projects involve only simulation on computers to demonstrate proof of principle. Many of them can benefit significantly by utility involvement to demonstrate their usefulness in nuclear power plants. Some of them will require extensive testing in a training or engineering simulator of a nuclear power plant prior to implementation in nuclear power plants. For instance, the use of neural networks to identify abnormal conditions or transients in nuclear power plants would require extensive use of a sophisticated nuclear power plant simulator, preferably a full-fidelity simulator of a particular nuclear power plant.

Identification of system Transients. Let us look at a typical application of neural networks, the identification of system transients in a nuclear power plant. The goal is the demonstration of the feasibility of using a neural network to diagnose different fault conditions or transients in a nuclear power plant through the use of pattern recognition. The initial task was the simulation of transients of a steam generator of a pressurized water reactor. For demonstration purposes, a set of data from a simulation of an isolated U-tube steam generator (UTSG) is used as the training data for the neural network. six step-change perturbations were introduced into the system, and four variables (i.e., sensor outputs or instrument readings) were chosen as the system responses to represent the system behavior for each perturbation. The time-records of these four quantities show that the patterns are quite different for each of the six perturbations listed above. Since the back-propagation network can be trained to distinguish between the different patterns, it can distinguish between the different kinds of steam generator perturbations. Subsequently, the number of variables used was reduced to three and then to two, and the neural network was still able to identify the transients.

In this study, a three layer back-propagation network was set up with 40 processing elements (PEs) in the input layer and three PEs in output layer to match the dimensions of the training input and output vectors. The middle layer contained $12 \mathrm{PEs}$ because this size

represented a reasonable compromise between ease of training and precision for the number of inputs and outputs. After 500 data training cycles using the back-propagation learning algorithm (which may require anywhere from a few seconds to a few hours, depending on the computer hardware and software used), the network readily distinguished between the six different perturbations. Random fluctuations were introduced into the trained network, ind the recall results showed that the network could still identify each of the UTSG transients correctly, even when the amplitude of the noise was equal to $90 \%$ of the amplitude of the signals. 
Modeling and Diagnostics in Nuclear Power Plants. This project has as its goal the development of diagnostic methods using neural networks to detect faults and transients in nuclear power plants. In some cases, this will involve the development of non-structured, nonalgorithmic models of process or major components using neural network techniques. In other cases, this model will be part of the knowledge base of an expert system.

A demonstration system was developed in which the knowledge base of an expert system communicated with a neural network in order to make recommendations to nuclear power plant personnel about the safety status of the plant when one or more components were removed from cervice. PRISIM, a computer program developed by JBF Associates for the Nuclear Regulatory Commission (based on the PRA of Arkansas Nuclear One) was used as the input to a neural network. The network was then trained to evaluate the increase in core melt probability associated with the removal of components or systems from service (as evaluated by PRISIM). Using a "constant risk" criteria, the expert system then advises the operator on actions to be taken to avoid violation of technical specifications and limiting conditions of operation.

Neuro-Connectionist Learning Techniques, Linear Generalization and spplicatic networks is being developed using a procedure known as "simulated condensation." Simulated condensation is a stochastic method of finding the optimum weights for layered feed-forward neural networks as an alternative to the commonly-used "backpropagation" of errors. Monte Carlo biasing techniques are used to speed the convergence of the learning process. Weight change selections are made from time-varying probability distributions, one for each weight. These distributions are then used to increase the probability of obtaining better results at the next weight selection. The problem is divided into several classes, and simulated condensation is used to optimize only one class of system parameters at a time. The procedure optimizes the non-threshold weights, the threshold weights, the learning coefficients, and the output coefficients separately. Hence, there is no need for an occasional uphill step, as is the case in the simulated annealing procedure. Simulated annealing is a faster optimization technique than back propagation or simulated annealing, but there is a loss of network generality. An internal report in draft form is attached to provide further information regarding the nature of the simulated condensation.

Neural Network Algorithms that "Learn" in a single cycle. A new approach to learning in neural networks has been developed that allows the network to learn to recognize a pattern in a single cycle. It uses competitive learning in the "hidden" layer and can learn as many patterns as there are processing elements in this layer. Both unidirectional and bi-directional versions of this algorithm have been demonstrated. A disclosure of this work is being submitted to the DOE as review for possible patents. Papers being submitted to DOE for patent review by zhichao Guo and Robert E. Uhrig are entitled: 
"A New Once-Through Learning Neural Network," and

"A Bi-Directional Once-Through Learning Neural Network,"

The following publications and internal reports were prepared from the work performed on this task and are included as appendices of this report.

R. E. Uhrig, "Use of Neural Networks in Nuclear Power Plants," Proceedings of the 7th Power Plant Dynamics, Control \& Testing Symposium, vol. 1, pp 26.01-26.05, Knoxville, Tennessee, May 15-17, 1989.

R. E. Unrig, "Use of Neural Networks in Nuclear Power Plant Diagnostics," Proceedings of the "International conference on Availability Improvements in Nuclear Power Plants," Madrid, Spain, April 10-14, 1989, (In press) 1989.

R. E. Uhrig and $z$. Guo, "Use of Neural Networks in Expert Systems for Nuclear Power Plant Diagnostics," Proceedings of "Applications of Artificial Intelligence VII," SPIE Technical symposium on Aerospace Sensing, Orlando, Florida, March 28-30, 1989.

E. B. Bartlett, "Neuro-Connectionist Learning Techniques, Learning Generalization, and Applications," UTK Internal Report, June 1989.

Zhichao Guo, "Use of Neural Network in an Expert System to Enhance Nuclear Power Safety." UTK Internal Report, April 1989.

\section{TASK 5: KNOWLEDGE ACOUISITION}

The primary purpose of this task is to develop psychological methods of extracting information or heuristic knowledge from experts for use in knowledge bases of expert systems. One cognitive psychology theory defines an expert as a person who has gathered some 50,000 "chunks" of knowledge on a particular subject over a ten-year period of time. (A "chunk" of knowledge is an understanding of a situation, system or concept to the extent necessary that the expert can use it to solve problems.)

Extracting knowledge from an expert is perhaps the "weak Iink" in the development of expert systems. Although most experts are cooperative with those building expert systems based on their knowledge, most experts redily don't know how they solve problems. They know the information and they can solve problems, but they cannot relate the two in an understandable way. Hence, it is the fob of the "Jnowledge engineer" to work with the expert to codify the expert's knowledge and put it in a form compatible with the knowledge base format. 
Individual Expertise. In extracting knowledge from a single expert a phenomenological interviewing style is used where the emphasis is on eliciting a train of thought from the person being interviewed and then translating it into rules (or other knowledge representation) for the expert system. In practice, this is done by first becoming familiar with the domain of the expert to make the expert feel he can talk to the interviewer on a technical level. The interviewer's job is to facilitate the expert's thoughts without leading him, interrupting him unnecessarily, or uning preconceived ideas of their own to categorize his thoughts.

Once the interview is complete, the information is translated into formal logic rules using principles of cognitive peychology. The focus is on connectionism, the concept that an idea is inked to another idea or set of ideas. The format provides a sormat of interrelated rules, not just a series of individual rules. The next step is to build a prototype system. The expert is brought back to test and critique the program. Since the knowledge base is totally separate from the inference engine, the latter can be modified until the expert agrees that the results of consultation with the expert system produces the same result as the expert in virtually every situation. Once up and running, the expert system still requires maintenance to keep it current, purge it of mistakes, and remove obsolete information.

Group Expertise. In a situation with group expertise, the group is surveyed to get a consensus opinion as to the course of action each rule should take. In programming this system, a prototype is made based on "common knowledge" rules. A group of knowledgeable individuals are surveyed, and the system is refined, based on those results. The system is "finished" when the majority of the expert group feels it captures the task or tasks from a psychological standpoint. It required an anticipation of how rules will be used, but still the open-mindedness needed for change. Non-verbal experts (usually printed sources of information) are still subject to the laws of connectionism and rules can be made to tie ideas together.

Community Expertise. Community expertise arises in a situation where the knowledge or expertise is distributed among several individuals (who probably have no regular interaction and may or may not be experts). This involves chaining together the expertise of several individuals to arrive at a decision. In example of community expertise in the nuclear power field is the review of proposed operational changes in a plant by a safety committee or board. Bach individual may be an expert in a specific field that pertains to the plant (e.g., heat transfer, fluld flow, neutronics, materials, radiological safety, or water chemistry). Learning how such a committee arrives at a decision could provide insight to the review process and allow the transfer of some of this review process to an expert system.

Results. the focus of contract) by

It is the area of "community expertise" that has become this task recently. Earlier work (prior to the DOE the principal investigator and his graduate student 
involved designing and constructing an expert system to recommend a restaurant for dinner. The knowledge base for this expert system was community expertise collected from many individuals at random "who were considered enough of an "expert" in the narrow field of restaurant selection). It is the development of techniques of extracting expertise from such a group that may become the focus of this project. Specifically, nuclear safety groups have varied backgrounds which they bring to bear on the safety problems of a apecific nuclear reactor. Developing techniques of extracting a better understanding of how safety decisions are reached could be very valuable.

It was the intent of this task to extend the earlier work to the reviews performed by a TVA safety group. Unfortunately, the recent reduction in force has created a situation that is not conduciva to the types of interactions required for this task. Reluctantly, it has been decided to terminate this Task at the end of the current contract on August 31, 1989. A substitute task will be proposed for FY-90.

\section{TASK 6: JOINT UT/TVA PROJECTS FOR NUCLEAR OPERATIONS}

In a nuclear facility, modifications, tests, and experiments are a part of life. However, in a licensed facility, these activities are bounded by Federal Regulations. Title 10 of the code of Federal Regulations Part 50 section 59 allows the licensee to modify the facility and perform test and experiments without prior Nuclear Regulatory Commission (NRC) approval, providing that the proposed activity meets certain requirements. While the requirements in 10CFR50.59 are very specific, the interpretation and implementation of these requirements may vary. In determining if an activity meets these requirements, the activities must be evaluated to determine their effects on the facility's safety Analysis. Not only are these evaluations very time consuming but they usually cross the lines of traditional discipline expertise. Also, experience has shown that these evaluations can vary in their conclusions and any modifications improperly evaluated could invalidate calculations used in the Design Basis. It would therefore be advantageous to have a syster that guides the evaluator in considering all possible effects of a proposed modification. At present, TVA performs a screening Review of each proposed activity to determine if a full scale safety Evaluation is appropriate. The proposed safety Review Advisor (SRA) will assist in the screening Review process.

The goal for this expert system is to direct the reviewer's attention to all the appropriate issues involved in evaluating the proposed modifications. The expert system under development will prompt the reviewer with a series of questions inguiring the nature of the proposed activity, the safety Related system being affected, and a final series of "Have you considered ...... effect?" questions. This final set of questions are multi-disciplined in scope, and include an 
appropriate set of reference listings from FSARs and Technical specifications for each question.

The examination of NRC and Industry documentation on the performance of 10 CFR50.59 reviews was to assure that all individuals working on the project were thoroughly familiar with all the issues surrounding a 50.59 review. The list of documents examined is not included in this report but is available upon request. Attendance of TVA training seminars was arranged for all personnel directly associated with this project. These seminars are used for the training of TVA engineers and managers to qualify them to perform the appropriate Screening and 50.59 Reviews.

Interviews with TVA employees who had recognized "expertise" in the performance of 10 CFR50.59 Reviews were conducted in order to ascertain a "best approach" for the Safety Review Advisor. As stated earlier, discussions with several individuals determined that the safety Review Advisor should act as an aid in performing screening Reviews. It was also decided that a prototype SRA should be constructed to test the approach chosen.

Research Goals. The SRA is organized according to specific plant systems such as Aux Feedwater supply, Emergency On-site Power Supply, etc. Obviously, there are very few systems in a nuclear facility that do not depend on other systems for support. Where these interconnections occur, there will also be appropriate logic interconnections between sections in the SRA. For the construction of the prototype SRA, the Emergency on-site AC Power supply system was selected. Work on the construction of the prototype is in its early phase. A study of the system by project personnel, and discussions with TVA engineers knowledgeable of the system are underway.

The Safety Review Advisor (SRA) is a prototype system designed to test the feasibility of utilizing expert systems to aid in the performance of 10CFR50.59 evaluations. Performing these evaluations is a complex task that requires a significant amount of knowledge about a nuclear plant and its safety related systems. The process requires that the engineer performing the evaluation be knowledgeable not only in several engineering disciplines but also the licensing documents.

The prototype SRA is designed to address all of the issues involved in performing proposed modifications to the standby Power systam at the sequoyah Nuclear Plant. The goal of the system is to direct the reviewer's attention to all the safety related questions that could be raised by modifying an existing plant system or component.

Results. During the first six months, significant progress has been made on the development of a prototype. This progress has occurred in three main areas. These areas are: 1) development of the prototype 
2) studying the feasibility of developing a full-scale SRA, including system characteristics and specifications, and, 3) the publication of a paper describing this task.

The development of the SRA is approximately $1 / 4$ to $1 / 3$ complete. To date, we have held several interviewe with the diesel generator expert in an attempt to incorporate his expertise into the systen. The eystem has been reviewed by him and the incorporation of his coments and suggestions is underway. These intarviows have allowed us to nodel all of the mechanical aspects of the diesel generator. Efforts are underway to arrange interviows with the experts in the electrical portion of the standby Power systen. We are also attenpting to arrange additional interviews with individuals who perform safety evaluations to incorporate their input.

Fessibility of Building a pull-scale system. The current version of the SRA was coded in Texas Instrument's PC PIus and runs on an IBY PC AT or compatible machine. PC Plus is a very powerful expert system development tool, however, some of its limitations raise particular concern as they relate to the project.

It has been determined that the ability to include plant schematics with the Safety Review Advisor is of particular importance because drawings can reduce ambiguity. Since the graphic capabilities provided by PC Plus are very 1 imited, the images could be generated using third party software (CAD/CAM) and interfaced with the prototype. We are currently surveying graphic packages and investigating the feasibility of interfacing these with PC Plus.

Another area of concern is efficiency. A full-scale implementation of the system under PC Plus, will definitely not execute at acceptable and satisfactory speed. Not only will the size of the knowledge base contribute to the deterioration of performance, but also the loading and execution of the graphic files. This problem is not very apparent while running the prototype but has been observed in knowledge bases of smaller size than the one projected for the full-scale system. Wa have estimated that a full-scale implementation of the sRA will contain at least 10,000 rules.

Finally, PC Plus does not offer a convenient mechanism to explore alternative path of reasoning during a consultation. Reviowere have indicated that a useful enhancement to future versions of the system would be the capability to examine nore than one case at a tine, without being forced to reload the knowledge base every tine (and therefore 1008 all input data). Another important euggestion, a generalization of the one mentioned above, is to provide a bechanier for saving and retrieving input data. With this nechanion, data from incomplete consultations could also be ade avallable. 
Publications. To date, the research efforts have been documented in two papers and an internal report that are included as appondices to this report. The first paper received the "OUTSTANDING PAPER AWARD" from the American Nuclear Society Eastern Regional student Conference.

"The Safety Review Advisor: An Expert system to Evaluate Proposed Modiflcations to Nuclear Power Plants. "James A. Boshers and Inrael F. Alguindigue, (Summary only), American Nuclear society Eastern Rogional student Conference, Knoxvilie, M, March 25, 1989.

"Safety Review Advisor," J. A. Bochers, I. E. Alguindigue, C. G. Burnett, and R. E. Uhrig, EPRI Conference on Expert systens, Orlando, FL, June 5-8, 1989.

J. A. Boshers, "SAFETY REVIEW ADVISOR: An Expert system to Ald Performance of 10CFR50.59 Evaluations," Internal Report, May 1989.

\section{CONCLUSION}

During FY-89, this program has made extraordinary progress in exploring several methods by which artificial intelligence, specifically expert systems and especially neural networks, can be utilized to address problems that arise in the operation of nuclear power plants. Most of this work has been exploratory in nature and not directed at producing software or hardware for direct use in plants. Rather, the program has been directed towards the goal of gaining a better understanding of the processes involved, improving them, and demonstrating the feasibility of applying them to nuclear power plants.

All but one of the tasks will be continued in FY-90, and additional tasks and subtasks will be undertaken. Our intent is to build on and be guided in our future work by the results obtained during FY-89. One of our primary goals continues to produce well-trained graduates with expertise in the artificial intelligence field who are motivated to work in the nuclear power field. Although there are no graduates whose M.S. thesis or Ph.D. thesis is the direct result of the research supported by this program, we expect that several such students will graduate during FY-90. It is our intent to transfer the technology developed in this program through our graduates, as well as by the conventional means of presentations and publications.

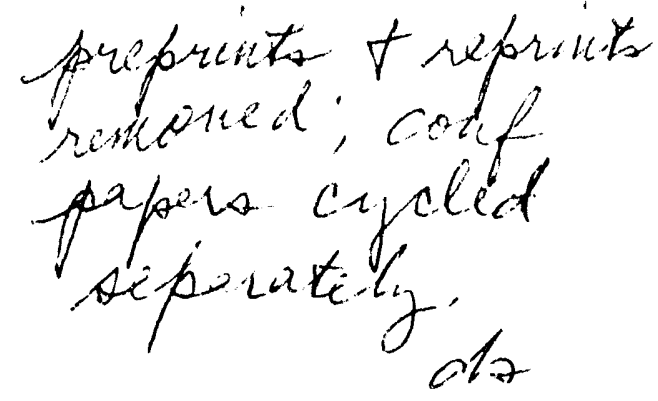



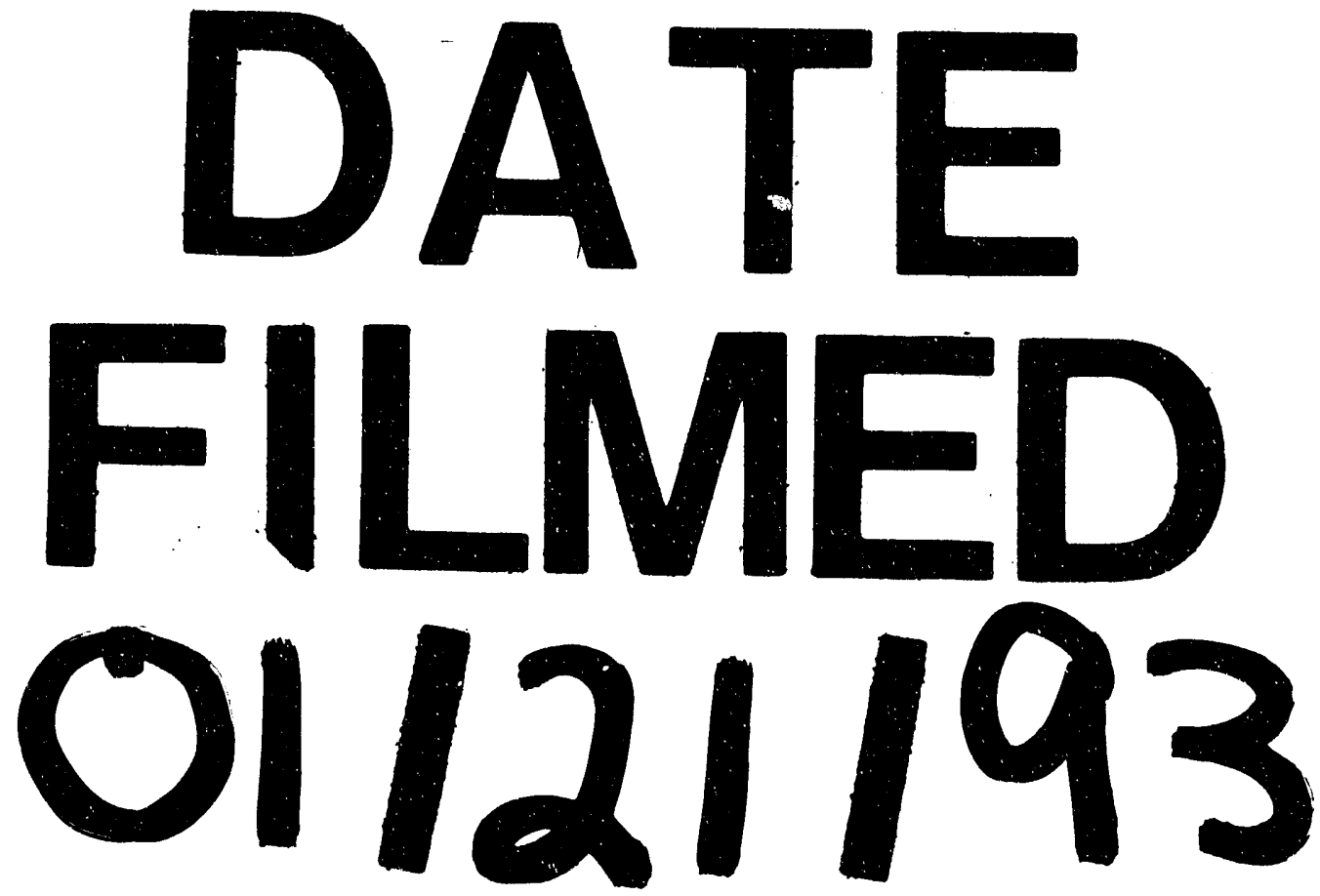
